\title{
Wie Gentests die Diabetestherapie verbessern können
}

\author{
Die Pharmakogenetik könnte helfen, die Diabetes- \\ therapie erfolgreicher zu machen. Schon heute las- \\ sen sich Patienten identifizieren, bei denen sich \\ Therapieversuche mit Metformin oder Inkretinen \\ eher nicht lohnen. Marktreif ist aber noch kein Test.
}

Die Pharmakogenetik soll letztlich die ärztliche Entscheidung unterstützen, welche Therapie im Einzelfall die besten Erfolgschancen hat. Außerdem hofft man, schon im Vorfeld der Behandlung abschätzen zu können, wie ein Patient das Medikament vertragen wird. Dazu ist auch ein verfeinertes Wissen über die pathophysiologischen Prozesse hinter dem Krankheitsbild erforderlich. Denn das individuelle "genetische Make-up“ eines Patienten bestimmt die Wirkung eines Arzneimittels entscheidend mit.

„Der Typ-2-Diabetes ist eine heterogene Erkrankung, zu der weit mehr gehört als nur ein erhöhter Blutzucker", betonte Prof. Jose C. Florez, Johns Hopkins University, Boston. „Allein an der Entstehung der Hyperglykämie sind bereits multiple Mechanismen beteiligt, die pharmakologisch angegangen werden können." Die erste Kernfrage ist: Lassen sich anhand der genetischen Disposition Voraussagen treffen, ob ein Wirkstoff oder eine Substanzklasse bei einem bestimmten Patienten wirken wird? Ein aktuelles Beispiel ist rs7202877, ein Gen-Locus in der Nähe des Chymotrypsin-Gens CTRB1/2, der mit einem erhöhten Diabetesrisiko in Verbindung gebracht wird.

\section{Chymotrypsin beeinflusst die Reaktion auf DPP-4-Hemmer}

Eine internationale Arbeitsgruppe um Leen M. $t^{\prime}$ Hart von der Universität Leiden hatte durch den Abgleich von genetischen Informationen und Patientenakten drei Gen-Loci identifiziert darunter auch den rs7202877-, deren Kodierung starke Auswirkungen auf den Effekt des Inkretins GLP-1 auf die Betazellen hat [1].

Inkretine werden im Darm sezerniert als Antwort auf eine kohlenhydrathaltige Mahlzeit und stimulieren die Insulinsekretion. rs7202877 steigert die Chymotrypsin-Aktivität, was zur Folge hat, dass die Betazellen empfindlicher auf GLP-1 ansprechen, die postprandiale Insulinausschüttung also zunimmt und das Risiko sinkt, einen Typ-2-Diabetes zu entwickeln. Zugleich nimmt die Response auf DPP-4-Inhibitoren ab, die den GLP1-Abbau unterbinden. „Die Mechanismen, die hinter den Wirkungen von Chymotrypsin auf die Betazellen und die DPP4-Wirkungen stecken, verstehen wir noch nicht richtig“, so Florez. Aber sie sind offenbar praktisch bedeutsam: Die Forscher konnten zeigen, dass Patienten, die am rs7202877-Locus ein GAllel tragen, deutlich schlechter auf einen DPP-4-Hemmer ansprechen als Probanden ohne G-Allel.

rs7202877 wäre also ein Kandidat für einen Gentest vor einer geplanten DPP-4-Hemmer-Therapie. GLP-1-Analoga sind hiervon übrigens nicht berührt, sie funktionieren bei G-Allel-Trägern ebenso wie bei Patienten ohne G-Allel.

\section{Neonataler Diabetes: Betazellen im Wintertschlaf}

Ein weiteres Beispiel ist der neonatale Diabetes, der lange als Form des Typ-1-Diabetes missdeutet wurde. Tatsächlich liegt hier aber keine autoimmune Betazell-Zerstörung vor. Die Betazellen befinden sich lediglich in einer Art Winterschlaf, weil ihnen Oberflächenstrukturen fehlen, welche die Insulinantwort auf Glukose auslösen.

Betroffene Patienten entwickeln schon in den ersten Lebensmonaten eine ausgeprägte Hyperglykämie. Phänotypisch sind sie von „echten“ Typ-1-Diabetikern nicht zu unterscheiden. Gibt man ihnen aber einen Sulfonylharnstoff, erwachen die Betazellen plötzlich zum Leben und sezernieren Insulin - nicht als Antwort auf Glukose, sondern auf das Arzneimittel. „Das ist ein klares Beispiel dafür, dass Pharmakogenetik einen großen Unterschied machen kann, nicht nur hinsichtlich der Versorgungsqualität, sondern auch der Lebensqualität“, betonte Florez.

Auf den Prüfstand gehört womöglich auch die gängige Empfehlung, die orale Therapie bei allen Typ-2-Diabetikern mit Metformin zu beginnen, solange keine Kontraindikation dagegensteht. Die Response auf Metformin variiert interindividuell nämlich beträchtlich.

\section{Metformin-Initialtherapie wirklich für alle Typ-2-Diabetiker gut?}

In einer Substudie des Diabetes Prevention Program wurde untersucht, inwieweit ein bestimmter Polymorphismus im Metformin-Transporter MATE1 zu diesen Unterschieden beitragen kann. Es stellte sich heraus, dass Metformin bei Teilnehmern mit den Genotypen CT und TT die Entwicklung eines Typ2-Diabetes verhindern konnte, nicht aber bei Probanden mit CC-Genotyp.

Auch die Intoleranz gegen Metformin, die etwa 10\% der Typ2-Diabetiker betrifft, hat offenbar genetische Grundlagen, nämlich Variationen im Organischen Cationen-Transporter 1 (OCT1). Nehmen Typ-2-Diabetiker Arzneimittel, die diesen Transporter hemmen wie beispielsweise Omeprazol oder Verapamil, steigt ihre Empfindlichkeit für gastrointestinale Nebenwirkungen von Metformin. Auch hier wurden bereits Allele identifiziert, welche die OCT1-Funktion reduzieren. Kommen OCT1-inhibierende Arzneimittel und Genvariante zusammen, vervierfacht sich die Intoleranzwahrscheinlichkeit. „Wenn wir solche Information vor Behandlungsbeginn haben, können wir 6-12 Monate frustranen Therapieversuch sparen und gleich mit einem Wirkstoff der zweiten Linie beginnen“, so Florez.

Es gibt also bereits eine Reihe von Ansätzen, pharmakogenetische Methoden zur Prädiktion von Therapieansprechen und Nebenwirkungen zu nutzen. Ob sie sich in der Praxis bewähren, müssen prospektive Studien belegen.

Manuela Arand

\footnotetext{
Literatur:

1. t'Hart LM et al. The CTRB1/2 Locus Affects Diabetes Susceptibility and Treatment via the Incretin Pathway. Diabetes 2013; 62: 3275-81.
} 